University of Nebraska - Lincoln

DigitalCommons@University of Nebraska - Lincoln

Faculty Publications, Department of Physics and Astronomy

1994

Rare-gas optics-free stable extreme-ultraviolet photon spectrometer for solar system studies

Melvin D. Daybell

Michael A. Gruntman

Darrell L. Judge

James A. R. Samson

Follow this and additional works at: https://digitalcommons.unl.edu/physicsfacpub

This Article is brought to you for free and open access by the Research Papers in Physics and Astronomy at DigitalCommons@University of Nebraska - Lincoln. It has been accepted for inclusion in Faculty Publications, Department of Physics and Astronomy by an authorized administrator of DigitalCommons@University of Nebraska - Lincoln. 


\section{Rare-gas optics-free stable extreme-ultraviolet photon spectrometer for solar system studies}

\author{
Melvin D. Daybell \\ Michael A. Gruntman \\ Darrell L. Judge \\ University of Southern California \\ Space Sciences Center \\ Los Angeles, California 90089-1341 \\ James A. R. Samson \\ University of Nebraska \\ Department of Physics \\ Lincoln, Nebraska 68588-0111
}

\begin{abstract}
We have developed a prototype spectrometer for space applications that require long-term stable EUV photon flux measurements. In this recently developed spectrometer, the energy spectrum of the incoming photons is transformed directly into an electron energy spectrum by taking advantage of the photoelectric effect in one of several rare gases at low pressures. Using an electron energy spectrometer operating at a few electron volts, and followed by an electron multiplying detector, pulses due to individual electrons are counted. The overall efficiency of this process is essentially independent of gain drifts in the signal path, and the secular degradation of optical components that is often a problem in other techniques is avoided.
\end{abstract}

Subject terms: atmospheric remote sensing; EUV spectrometers; space instrumentation.

Optical Engineering 33(2), 445-450 (February 1994).

\section{Introduction}

Research programs within planetary, cometary, and heliospheric sciences ultimately require absolute solar flux data in the highly variable EUV spectral region. The scientific programs have matured to the point where flux estimates that currently may be wrong by a factor of 2 or more are no longer acceptable. Both planned planetary observations and ongoing missions require absolute solar flux data that have not been compromised by degradable optics and detectors. Despite myriads of observations, the absolute solar UV flux and its variation throughout the wavelength region below $1000 \AA$ have not been well established for detailed aeronomical and astrophysical studies. The susceptibility of previously flown solar instrumentation to solar-driven degradation of both the optics and detectors makes it difficult to separate true solar variability from changes in the instrumentation sensitivity.

The goal of this work is to present an alternative way to measure the solar EUV spectrum, a method with a minimum of degradable optical (hence, "optics-free") elements. Such an approach, described by Judge, Ogawa, and Gruntman, ${ }^{1}$ should eventually result in the development of a new generation of highly stable and reliable spectrometers for monitoring the solar EUV radiation spectrum on a continuous

Paper MI-040 received April 1, 1993; revised manuscript received Aug. 19, 1993; accepted for publication Aug. 30, 1993. This paper is a revision of a paper presented at the SPIE conference on Instrumentation for Planetary and Terrestrial Atmospheric Remote Sensing, July 1992, San Diego, Calif. The paper presented there appears (unrefereed) in SPIE Proceedings Vol. 1745

(c) 1994 Society of Photo-Optical Instrumentation Engineers. 0091-3286/94/\$6.00. basis. The idea of the technique was first proposed by Samson $^{2}$ and uses photoionization of gas target atoms to convert an EUV photon spectral distribution into an energy distribution of the photoelectrons, which is finally measured. Although this technique has been applied in laboratory studies, the feasibility of its implementation in space instrumentation has yet to be demonstrated. Specific conditions of space applications pose stringent requirements on instrument characteristics, particularly its speed and consumption of the expendable target gas.

Different approaches are possible to measure the photoelectron energy distribution. A photoelectron spectrometer based on measurement of the photoelectron current is hardly acceptable because of its low sensitivity. Attempts to increase photoelectron collection by introducing magnetic fields and using diffusion of electrons in the gas face serious problems of magnetic field inhomogeneities and electron inelastic collision processes. ${ }^{3}$ The overall weight of an instrument based on such an approach practically precludes its application in space. The only feasible way seems to be to use an electron energy analyzer and count individual electrons. ${ }^{1,4}$ To increase the instrument's sensitivity, and consequently its speed, continues to be a challenging task. Another challenge is to eliminate the stray electron and photon background count rate. This could be achieved by requiring coincident detection of the positive photo ions. The aim of this work is to give a comprehensive view of the photoelectron energy analysis technique, to demonstrate its feasibility as a photon spectrometer, and to discuss some inherent advantages and disadvantages of various ways of implementing it. 


\section{Optics-Free EUV Spectrometers}

The strategy of using a rare-gas optics-free absolute EUV photon spectrometer for solar system studies takes advantage of a novel technique first presented by Samson. ${ }^{2}$ Using this method, it became possible to monitor the absolute spectral irradiance of a UV light source and to determine accurately the values of molecular parameters requiring a knowledge of the absolute flux from such sources. This same capability to measure the absolute flux over periods longer than a year promises to be very useful in time and wavelength resolved EUV solar flux surveys below $1000 \AA$.

The optics-free spectrometer (OFS) instrument actually performs its spectroscopy on electrons using an electron energy analyzer (Fig. 1). This analyzer transfers the spectral distribution of the light source, via photoionization of a stable atomic gas (rare gas), into an electron energy distribution through the Einstein equation $h v=I+E_{e}$, where $h v$ is the energy of the photons, $I$ is the ionization potential of the raregas atom, and $E_{e}$ is the energy of the ejected photoelectrons. Thus, in the case of photoionization of helium, for example, a discrete electron energy is produced for each discrete wavelength emitted by the source. If the other rare gases are used, two discrete electron energy groups are produced for each discrete wavelength. These two groups correspond to the creation of the ion in its ${ }^{2} \mathrm{P}_{3 / 2}$ or ${ }^{2} \mathrm{P}_{1 / 2}$ state. Because a gas with a known and, more importantly, time-independent photoionization cross section is used, the analyzer is capable of providing stable values of the absolute spectral irradiance of the light source. It is only necessary to maintain the geometry constant, know the gas pressure, and use a reliable electron counter, for example, one of the channeltrons or microchannel plate assemblies of the type used in earlier long-duration space missions.

The principle of operation is as follows: The number of electrons detected per second $N(\lambda)$ depends on the number of photons per second $J A$ passing through the effective area $A$ of the ionization cell visible to the electron energy analyzer, the effective thickness $t$ of the cell, the number density $n$ of the target gas, its photoionization cross section $\sigma$ for the angle where the analyzer's entrance aperture is located, and the solid angle $\Delta \Omega$ subtended by this aperture at the cell. An additional factor $\eta$ describes the transmission of the electron analyzer and the efficiency of the detector.

Then,

$N(\lambda)=J A \sigma n t \Delta \Omega \eta$

This can be expressed as

$N(\lambda)=C(\lambda) J(\lambda) \sigma(\lambda) n$,

where

$C(\lambda)=A t \Delta \Omega \eta$

is an instrumental constant that can be calculated from the geometry or, more often, determined in a calibration measurement. If the absolute radiant energy of the source used in the calibration is known, this measurement will transform the OFS spectrometer into a secondary standard; if not, $C(\lambda)$ can be expressed in relative units. Later, we discuss an ionelectron coincidence counting technique that makes it pos-

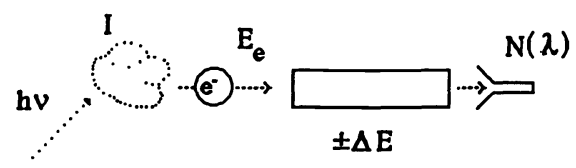

Fig. 1 The OFS concept.

sible to monitor the stability of $C(\lambda)$ after the experiment is in place.

A variety of designs exist for electron energy analyzers. The requirements here differ from those widely used in EUV and $x$-ray surface studies, solid state physics research, electron microscopy, and chemical analysis in that we have a large uniform and diffuse source, but a high collection efficiency for electrons produced in the source volume is still required. The electron flux is low, so that space charge effects can be ignored. The energy resolution required is not excessive. We do, however, have to scan more than one decade of electron energy and must usually work in the presence of an unavoidable background of neutral rare-gas atoms that come through the exit slit of the photoionization cell along with the electrons.

A schematic view of our laboratory OFS is shown in Fig. 2. The EUV photons enter from the right through a series of slits ahead of a small ionization volume, or gas box, located above an electron lens. Behind the gas box, on the left, is a light trap. The ion-coincidence accessory above the gas box will be described later. The flow of target gas out of the gas box region is constrained by the slits along the incoming photon beam and by the small slit on the bottom of the gas box connecting it with the electron lens. The gas pressure is 10 to 50 mTorr and gas consumption is small. The electron lens allows escaping target gas to be removed from the path of the electrons being analyzed.

Photoelectrons exiting the bottom slit are imaged on the entrance slit of a 180-deg hemispherical plate electrostatic energy analyzer, and electrons of the chosen energy come to a focus at its exit slit. After passing through this slit they are detected by a channeltron-style electron multiplier.

The electron energy spectrum is obtained by sweeping the potentials applied to the lens-analyzer system and monitoring the count rate from the channeltron.

\section{Performance of Some Simple Optics-Free Spectrometers}

Two emission spectra obtained with our OFS are shown in Figs. 3 and 4. The simplest emission spectrum is that of atomic helium shown in Fig. 3 . Here, the $n=2 \rightarrow 1(584 \AA)$, $3 \rightarrow 1(537 \AA)$, and $4 \rightarrow 1(522 \AA)$ transitions are clearly visible. The more complex argon emission spectrum is shown in Fig. 4. Both spectra were obtained using argon as the target gas in which the electron-ion pairs were produced. In the helium spectrum, the 178-meV splitting associated with the two ionization states of the argon target is clearly resolved. (At 584 $\AA$, this splitting is equivalent to $4.9 \AA$. The resolution is clearly better than this.) The absence of a significant stray electron background should also be noted. We have included a $20 \times$ enlargement of this spectrum (Fig. 5). A typical helium spectrum obtained using a representative grating spectrometer is included for comparison (Fig. 6).

To reach the data rates required for shuttle applications, we are planning to add a microchannel plate position- 


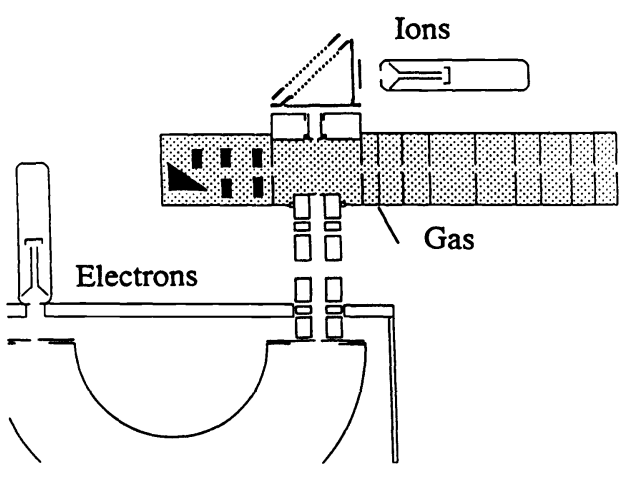

Fig. 2 Schematic diagram of an OFS using an electron lens, a spherical electrostatic electron energy analyzer, and a channeltron electron multiplier. Also included is an optional channel to detect the coincident photo ion.

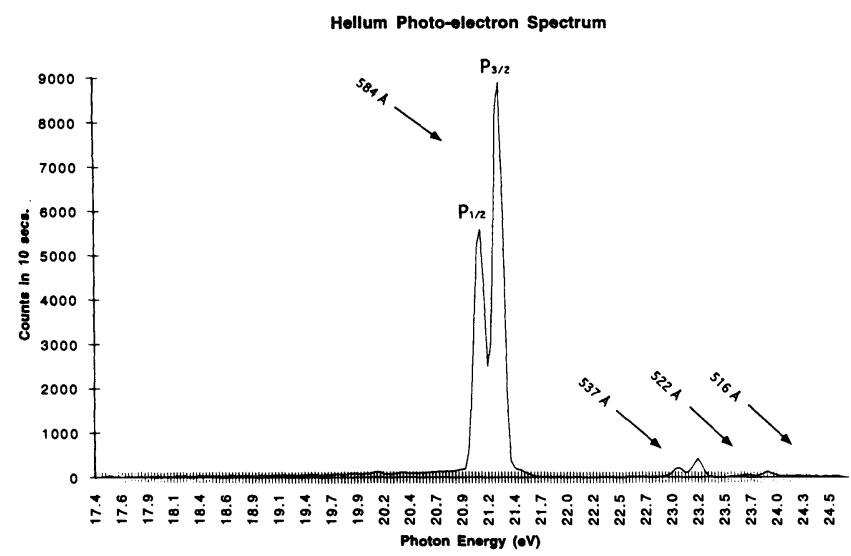

Fig. 3 Electron counts per energy step versus electron energy with an OFS similar to Fig. 2. The EUV source was an undispersed $\mathrm{He}-\mathrm{I}$ microwave glow discharge. The rare-gas ionization cell contained argon.

sensitive detector at the back focus of the electron energy analyzer, replacing the back slit and the channeltron. In essence, this yields many electron energy channels in parallel and increases the data acquisition rate in direct proportion to the effective number of channels, without increasing the consumption of target gas.

The ion coincidence channel shown above the gas box is an additional enhancement that lets us sweep out and detect the ion created in the same photoionization event that produced the electron. This optional feature allows us to eliminate background events and to monitor the absolute sensitivity of the OFS after it is in service. Implementation of this enhancement will improve the already high quality data shown in Figs. 3 and 4.

One component not shown in the schematic is the magnetic shielding used to screen out the Earth's field. Absence of this shielding is known to restrict the wavelength region of OFS operation to shortward of around $400 \AA$. We hold the field in our spectrometer to about $1 \mathrm{mG}$ and can operate throughout the rare-gas ionization region $(\lambda<1022 \AA)$.

Except for helium, each of the rare gases that might be used as a photoionization target may be left in one of two ionized states with nearly the same energy. This leads to a pair of photoelectron peaks for each incident photon wavelength, as seen in Fig. 3 for an argon target ionized by the

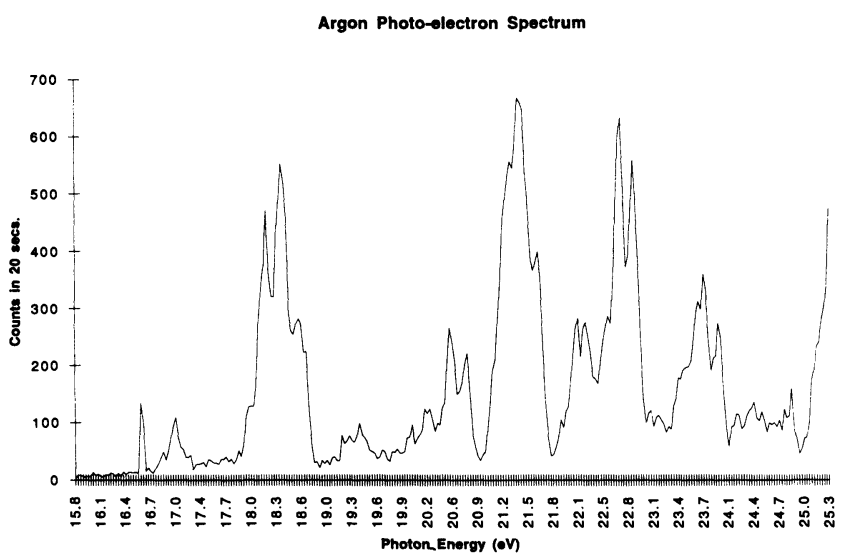

Fig. 4 An OFS spectrum of Ar II. The conditions were the same as for Fig. 3, except that an undispersed Ar-II microwave glow discharge was substituted for the $\mathrm{He}-\mathrm{I}$ source.

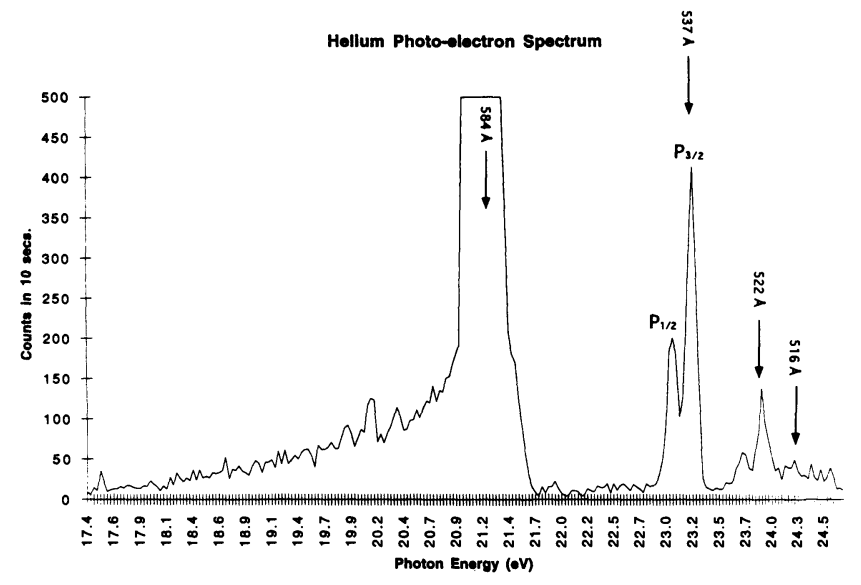

Fig. 5 The He-I OFS spectrum of Fig. 3 expanded vertically by a factor of $20 \times$ to better show the weaker elements of the He-I principal resonance series.

584- $\AA$ line from a helium lamp. Using an advanced deconvolution algorithm based on that of Allen and Grimm ${ }^{6}$ and taking the "instrument function"' to be the experimental response to the sharp isolated 584- $\AA$ line, it is possible to transform the output trace of the OFS into a very good replica of the input photon spectrum. This has been done in Figs. 7(a) and 7(b) at two different vertical scales for the resonance series of helium discussed earlier. Notice that the annoying line doubling in the output trace has disappeared. Also, the resolving power appears to have improved significantly.

Another appropriate use for deconvolution is to trade instrument sensitivity for resolving power electronically, as required. A photoelectron spectrometer with relatively high sensitivity, and therefore relatively poor resolution, can gain resolution by scanning through its range more slowly to accumulate the better electron counting statistics required for satisfactory performance of the resolution enhancing algorithm.

Some time ago one of us (Samson) obtained results similar to these with a different electron analyzer geometry, the cylindrical mirror analyzer (CMA), in which the photoelectrons 


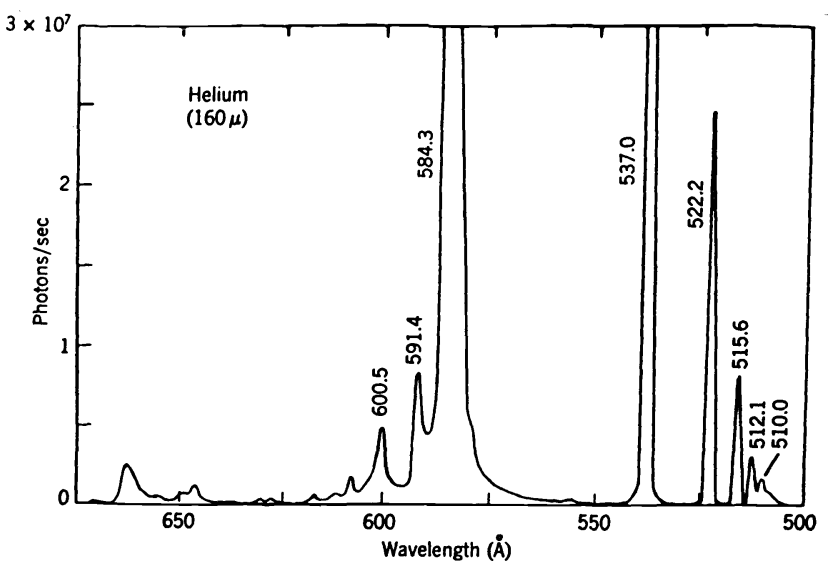

Fig. $6 \mathrm{~A} \mathrm{He}-\mathrm{I}$ spectrum obtained using an optical monochromator (the source was a dc glow discharge ${ }^{5}$ ).

created on the axis of a cylindrical capacitor pass outward through an annular slit in the inner plate of the capacitor and then are forced back toward a second identical slit further along the inner cylinder by an electrostatic field between the two plates to refocus on the axis. The results were similar, except that many more electrons were collected and the signal was stronger, but the background due to scattered electrons was more troublesome.

For space applications, it will be necessary to utilize OFS configurations that can gather EUV spectra in reasonable times (a half hour or less). An electron energy analyzer with a position-sensitive detector in its image plane (so that it becomes an electron spectrometer) can acquire data at many energies at once. The hemispherical electron energy analyzer appears to be able to utilize a position-sensitive detector more effectively than some of the other geometries and may be affected less by scattered electrons than the CMA. The scattered electron background present in our OFS (Fig. 5), which uses a hemispherical energy analyzer, is at least an order of magnitude less than in the CMA mentioned earlier.

Two modes are commonly used to scan the measured photoelectron energy over the desired spectral range. In the first mode, the potential difference between the capacitor plates of the analyzer is held constant, fixing the analyzer pass energy at 2 or $3 \mathrm{eV}$, and the electron energy is scanned by varying the potential difference between the rare gas ionization cell and the analyzer. This has the desirable effect of holding the energy resolution constant across the whole energy range, leading to excellent fractional energy resolution at high energies. On the other hand, the entrance slit of the electron analyzer combined with the housing of the ionization cell form a basic electron lens, which must obey the laws of electron optics that tell us that if the lens slows the electrons down, a smaller fraction of the photoelectrons leaving the object space of the lens will fit into the fixed acceptance solid angle of the analyzer; thus, the transmission of the system will drop as the potential retarding the electrons is increased. Another obvious approach is to keep this retarding potential constant, but scan the voltages on the capacitor plates of the analyzer. In this second mode, the fractional energy resolution is fixed, but the transmission at high energies is better. This trade-off is desirable in many space applications.

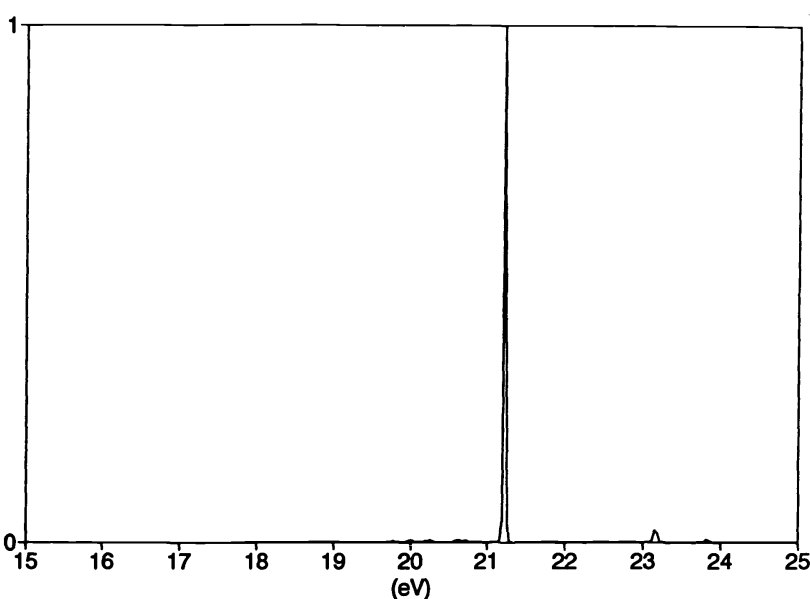

(a)

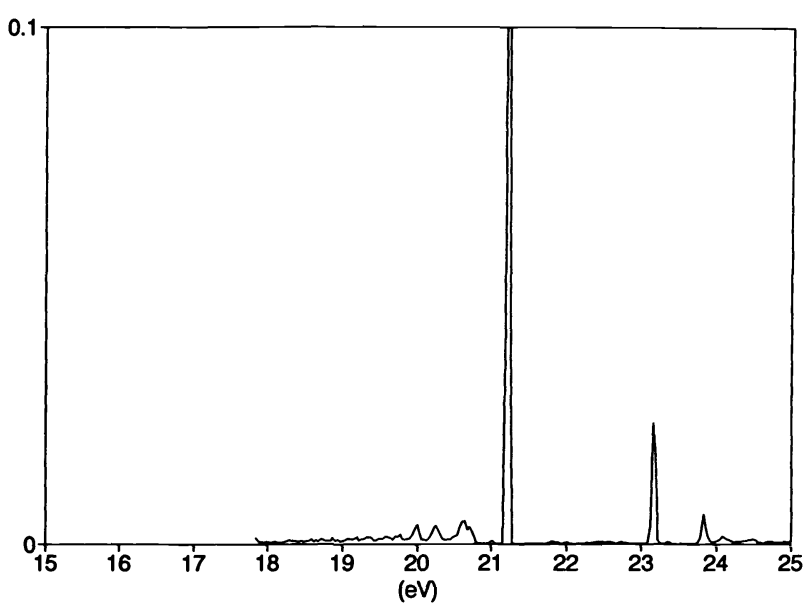

(b)

Fig. 7 (a) Deconvolution of the He-I OFS spectrum of Fig. 3 and (b) the same data expanded vertically $20 \times$ (compare to Figs. 5 and 6).

\section{Design Features of a Recent Optics-Free Spectrometer}

In choosing the design parameters for an OFS, it is helpful to notice that the mean free paths of photoelectrons and of atoms in a rare gas are roughly comparable, while that of the photons is considerably longer. Once we choose a gas pressure for our ionization cell, the dimension of the cell must be limited to a fraction of the electron mean free path if we are to get the photoelectrons out. This in turn limits the crosssectional area of the window the cell can open to the sun. Since the number of detectable photoelectrons produced depends on the product of the target gas pressure, the area of the photon beam, the size of the slit through which electrons exit the cell, and the acceptance solid angle of the electron analyzer, if we scale up all three dimensions of the spectrometer, keeping the solid angle constant and dropping the pressure to keep the mean free path in scale, the counting rate will scale approximately as the square of the dimensions. The loss rate of target gas out the window to the sun will increase only linearly with the scale, since we are in the molecular flow regime. A rough estimate indicates that a 
$100-\mathrm{cm}^{2}$ window would support several spectra per day for a year with a 1-L gas supply initially at a few hundred pounds per square inch. The resulting OFS system would be rather large. Our present system has a window of $0.2 \mathrm{~cm}^{2}$ and its analyzer is about $14 \mathrm{~cm}$ across.

One upper limit on the size of the analyzer is the value of the ambient magnetic field, which will reduce its resolution. As a rule of thumb, an analyzer with a resolution of $1 \%$, $10 \mathrm{~cm}$ across, can tolerate a field of about $10 \mathrm{mG}$. Simple Helmholtz coils or magnetic shielding can reduce local fields to this value.

Since the presence of gas atoms in the analyzer, and even more, in the detector degrades performance, an effort must be made to separate these atoms from the electrons as soon as possible using differential pumping. This is the main reason for introducing the electron lens shown in Fig. 2. The lens images the hole in the ionization cell onto the entrance slit of the electron analyzer while allowing atoms escaping from the same hole to be pumped away. The analyzer is nearly enclosed in its own box and may be separately pumped as necessary. With this system, the pressure in the ionization cell is limited by the mean free path of the electrons, or by the rate at which the vacuum pumps the gas out the long entrance tube provided for the photons.

Another function of the electron lens is to image an aperture stop in the object space to an image inside the electron energy analyzer, where it forms a virtual stop to reduce the number of stray electrons. The first and last electrodes of this lens are the ionization cell and the analyzer's entrance slit, respectively, and the lens can be used to scan the energy of the photoelectrons across the pass energy of the analyzer, when this is desirable. The pass energy can also be scanned and since all electrode potentials are interfaced to a CAMAC system, we have computer-controlled resolution as well as energy and can trade off resolution for counting rate as needed. The electron lens as well as the interior of the ionization cell and the electron analyzer have been coated with colloidal graphite dissolved in isopropanol to reduce contact potential effects and cut down on electron charging and stray light scattering.

An ionization-type pressure gauge based on radioactive tritium $^{7}$ is used to monitor the pressure in the photoionization cell. The pressure signal is read out by a digital electrometer. This design has served us well for many years.

A modular channeltron/high-voltage-supply/pulse amplifier (Amptektron, Amptek Inc., Bedford, Mass.) serves as the electron detector. A resistive anode behind a stack of microchannel plates forms a position-sensitive detector that can replace the Amptek unit when needed.

In spite of the remarkably good performance of the OFS elements examined so far, some problems remain that have encouraged us to look at an enhancement of the basic strategy. Some stray electron background is still present and it would be useful to have some way to check on the stability of the electron detector. It would also be possible to cover a wide range of photon wavelengths at once if a mixture of rare gases could be accommodated in the ionization cell. For example (see Table 1), a mixture of $\mathrm{He}$ and $\mathrm{Xe}$ would allow the whole range from 157 to $1022 \AA$ to be covered without complications from double photoionization.

Many of these challenges can be largely eliminated by implementing an ion coincidence technique that will now be
Table 1 Parameters of the rare gases.

\begin{tabular}{|c|c|c|c|c|c|c|}
\hline gas & shell & $Z$ & \multicolumn{2}{|c|}{$\begin{array}{c}\text { ionization potential } \\
(\mathrm{eV})\end{array}$} & \multicolumn{2}{|c|}{$\lambda_{\text {threatold }}(\AA)$} \\
\hline & & & single & double & single & double \\
\hline $\mathrm{He}$ & $1 \mathrm{~s}$ & 2 & 24.580 & 78.980 & 504.39 & 156.98 \\
$\mathrm{Ne}$ & $2 \mathrm{p}$ & 10 & 21.55 & 62.62 & 575.1 & 198.0 \\
$\mathrm{Ar}$ & $3 \mathrm{p}$ & 18 & 15.77 & 43.39 & 786.2 & 285.7 \\
$\mathrm{Kr}$ & $4 \mathrm{p}$ & 36 & 13.99 & 38.55 & 886.2 & 321.6 \\
$\mathrm{Xe}$ & $\mathrm{5p}$ & 54 & 12.13 & 33.3 & 1022 & 372 \\
\hline
\end{tabular}

described. In the center of the top of the gas box in Fig. 2 is an additional aperture insulated from the gas box. This aperture is cut in a disk forming the bottom of a short metal cylinder and permits the positive ion left behind by the photoelectric event described earlier to be detected. A large voltage pulse $(\sim 500 \mathrm{~V})$ can be imposed on this cylinder in such a way as to apply an impulse to the ion directed toward the aperture. This pulse will be triggered only by the arrival of a photoelectron at the electron detector discussed earlier, which will occur on the order of $100 \mathrm{~ns}$ after the photoionization event. Beyond the aperture, a constant electric field $(\sim 6 \mathrm{kV} / \mathrm{cm})$ accelerates the ion toward an electrostatic mirror (of wire grids), which reflects the ion into a detector (a channeltron is shown). A properly delayed coincidence between the output of the ion detector and that of the electron detector is required for a count to be considered valid. The electrostatic mirror serves to isolate the ion detector from the gas cell and to allow lower gas pressures at this detector, for which another Amptek module modified to collect positive ions can be used.

With this scheme, the coincidence counting rates will be lower than those described earlier, since some photo ions will be missed, and the gas target pressure will be lower with the ion aperture installed. But any change in the efficiency of either detector will show up as a relative change in the individual detectors' count rates and the coincidence rate, the stray electron background will be eliminated, and, by requiring different delay times between the coincidences, the time of flight of the ion can be selected, thus determining which species of rare-gas ion it is.

A little ingenuity may be required to apply the ion coincidence idea to a few of the most efficient OFS designs, but its potential virtues make it worth investigating for some applications and would lead to a well-characterized OFS well suited to providing highly reliable absolute flux data in the EUV spectral region.

\section{Acknowledgments}

This work is supported in part by NASA contracts NAGW-2354 and NAGW-2677.

\section{References}

1. D. L. Judge, H. S. Ogawa, and M. A. Gruntman, “An optics-free EUV spectrometer,' EOS Trans. AGU Fall Meeting Suppl. 73(43), 1486 (1990). See also M. D. Daybell, M. A. Gruntman, and D. L. Judge, "A rare gas optics-free EUV photon spectrometer for solar system studies," Proc. SPIE 1745, 149-155 (1992).

2. J. A. R. Samson, Proc. Intl. Symp. Synchrotron Radiation Users, Daresbury, U.K., 4-7 Jan. 1973.

3. E. D. Mischchenko, I. L. Rebo, and A. B. Utkin, "Effect of magneticfield inhomogeneities on the operation of a collisional photoionization spectrometer,', Opt. Spectrosc. (USSR) 62(5), 703-704 (1987).

4. J. S. Vickers, D. M. Cotton, T. A. Cook, and S. Chakrabarti, "Gas ionization solar spectral monitor," Proc. SPIE 1745, 132-139 (1992). 
5. J. A. R. Samson, Techniques of Vacuum Ultraviolet Spectroscopy, p. 141, Pied Publications, Lincoln, Nebraska (1967).

6. J. D. Allen and F. A. Grimm, "High-resolution photoelectron spectroscopy through deconvolution," Chem. Phys. Lett. 66, 72-78 (1979).

7. J. O. Maloy, R. W. Carlson, U. G. Hartmann, and D. L. Judge, "Rocket instrument for measurement of the solar $\lambda 584 \AA$ He I line," Space Sciences Center Technical Report, p. 2-12 and 2-13, Univ. of Southern California, Los Angeles (1974)

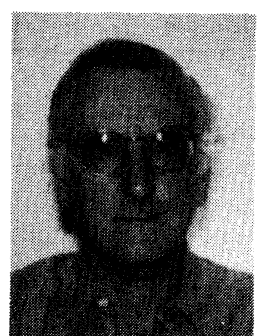

Melvin D. Daybell received a BS from New Mexico State University and a PhD in physics from the California Institute of Technology. He became a physics professor at the University of Southern California in 1968 and joined its Space Science Center in 1991 to develop the optics-free EUV spectrometer.

Michael A. Gruntman: Biography and photograph appear with the paper "Novel low-energy neutral atom imaging technique" in this issue.

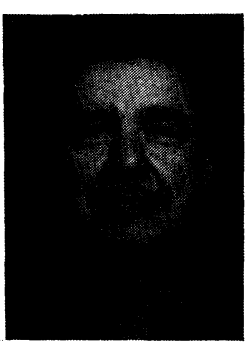

Darrell L. Judge received his $\mathrm{PhD}$ in physics from the University of Southern California (USC) in 1965. He has been an industrial and government consultant since 1960 and a physics professor at USC since 1965. Beginning in 1977 he has held the position of director of the USC Space Sciences Center. His primary research interests are in laboratory atomic and molecular physics and space physics.

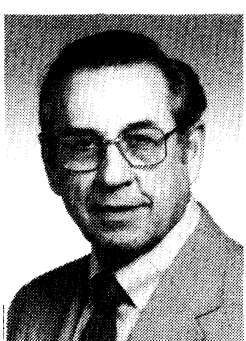

James A. R. Samson earned a BSc from Glasgow University and an MS and PhD from the University of Southern California. $\mathrm{He}$ is the author of numerous papers and a text dealing with basic atomic and molecular physics and the interaction of VUV radiation with matter and past editor of the Journal of the Optical Society of America. Currently he is Regents' Professor of Physics at the University of Nebraska. 